\title{
Fähigkeitsausweis Vertrauensarzt
}

\author{
J. Zollikofer
}

Die Ärztekammer hat dem Fähigkeitsausweis Vertrauensarzt zugestimmt. Ein altes Desideratum der FMH ist damit erfüllt. Der Anschlussvertrag an den Vertrauensarzt-Vertrag, in welchem santésuisse und FMH die Schweizerische Gesellschaft der Vertrauensärzte (SGV) mit der Umsetzung beauftragen, ist unterzeichnet. Der erste WB-Kurs für den Fähigkeitsausweis startet im Spätsommer 2003.

Korrespondenz:

Dr. med. Jürg Zollikofer

Präsident der Schweiz. Gesellschaft

der Vertrauensärzte

CH-8475 Ossingen

E-Mail: juerg.zollikofer@hin.ch

\section{Vertrauensarzt im KVG}

Art. 57 KVG regelt Stellung und Aufgaben der Vertrauensärztinnen und -ärzte (VA). Bis der Absatz 8 (Weiterbildung der VA und Regelung der Weitergabe der von Leistungserbringern erhaltenen Angaben an den Versicherer) in die Tat umgesetzt werden konnte, wurde fast so viel Zeit gebraucht wie für die Einführung des TARMED.

\section{Fähigkeitsausweis Vertrauensarzt}

Seit 1. Januar 2002 ist der Vertrauensarzt-Vertrag in Kraft. Ende April 2003 hat nach der Kommission für Weiter- und Fortbildung der FMH (KWFB) auch die Ärztekammer den Fähigkeitsausweis Vertrauensarzt genehmigt. Ende Mai haben nun santésuisse und FMH den Anschlussvertrag unterschrieben. Damit wird die Schweizerische Gesellschaft der Vertrauensärzte (SGV) mit der Umsetzung des Weiter- und Fortbildungskonzeptes beauftragt. Beide Dachverbände leisten hier wesentliche finanzielle Beiträge, was besonders betont werden muss, handelt es sich doch eigentlich um eine Aufgabe, für welche der Bund aufkommen müsste. Der VertrauensarztVertrag ist im Handbuch der Schweizerischen Krankenversicherung abgedruckt. Ein wesentlicher Punkt, die Ausdehnung des VA-Vertrages auf die Zusatzversicherungen, konnte leider vorderhand noch nicht verwirklicht werden, ist aber aus Sicht der SGV weiterhin anzustreben.

\section{Aufgaben der Vertrauensärzte und Vertrauensärztinnen}

Um den hohen Anforderungen gerecht werden zu können, müssen die Vertrauensärzte über eine breite Erfahrung aus der ambulanten und stationären Medizin sowie über fundierte Kenntnisse der relevanten Gesetze und Verordnungen verfügen. Die bisher lediglich geforderten 5 Jahre Praxis oder leitende Stellung an einem Spital konnten deshalb nicht mehr genügen. Bekanntlich fristet die Versicherungsmedizin an den Universitäten und in der Ausbildung während des Medizinstudiums ein Mauerblümchendasein. Im jetzt besiegelten Vertrag wurde deshalb nach einer Möglichkeit gesucht, diese Lücken im Wissen bezüglich Versicherungsmedizin zu schliessen. Das Curriculum im Fähigkeitsausweis Vertrauensarzt trägt dieser Tatsache Rechnung.

Die Kernaufgabe der Vertrauensärzte ist die Beratung der Versicherer bezüglich WZW: Wirksamkeit (nach wissenschaftlichen Methoden nachgewiesen), Zweckmässigkeit und Wirtschaftlichkeit von Leistungen. Dazu kommt noch der 4. Faktor: unangefochten. Damit sie dieser Aufgabe nachkommen können, haben ihnen die Leistungserbringer die für eine Beurteilung notwendigen Angaben zu liefern. Dazu gehören alle Akten, auf Verlangen also auch Zusammenfassungen der Krankengeschichte (Austrittsberichte) und Operationsberichte. $\mathrm{Zu}$ beachten ist, dass der Vertrauensarzt derjenige ist, der bestimmt, was ihm auszuhändigen ist und nicht der Leistungserbringer. Bei Bedarf können vom Auskunftspflichtigen einzelne Teile (z.B. für Beurteilung der Leistungspflicht nicht relevante anamnestische Angaben bei einer psychiatrischen Hospitalisation) abgedeckt werden. Einzig der Geheimnisherr, und das ist der Patient und nicht etwa der Leistungserbringer, hat das Recht, die Herausgabe von medizinischen Akten an den VA zu verweigern. Im Gegenzug gibt dies dem Versicherer das Recht, die Zahlung zu verweigern, da er die Sachlage (durch den VA) nicht prüfen (lassen) kann. Einfacher wird es sein, wenn der medizinische Akten herausgebende Leistungserbringer den VA in kritischen Fällen darauf aufmerksam macht, dass es sich um besonders heikle Angaben handelt, welche nur für den VA bestimmt sind.

Die Verantwortung für Verwendung und dann Archivierung liegt gemäss VA-Vertrag bei den Vertrauensärzten. Diese allein sind dafür verantwortlich, welche Angaben an die Leistungsabteilung des Versicherers weitergegeben und v. a. aber, wie die erhaltenen medizinischen Akten archiviert werden und wer dazu Zugang hat. 
Der 1. Weiterbildungskurs 2003 findet an folgenden Daten statt:

21.-23.8.

25. -27.9 .

23. -25.10 .

20.-22.11.

Die fachliche Leitung dieses Kurses liegt bei der KWFB der SGV.

Kosten für VA von Krankenversicherern Fr. 500.- pro Modul, SGV-Mitglieder Fr. 450.-.

Für nicht im KVG tätige Teilnehmende müssen die Bruttokosten verrechnet werden.

Interessenten melden sich bei der Geschäftsstelle der SGV c/o MBC Markus Bonelli Consulting, Wülflingerstrasse 59, 8400 Winterthur, Fax 0522226868 oder: markus.bonelli@bonelli.ch

\section{Weiterbildung}

Der 1. Weiterbildungskurs für den Fähigkeitsausweis Vertrauensarzt wird im Herbst 2003 stattfinden (siehe Kasten). Er umfasst 4 Module à je $2 \frac{1}{2}$ Tage mit folgenden Schwerpunkten: Sozialsystem, alle relevanten Gesetze und deren Verordnungen (z.B. Krankenpflegeleistungsverordnung, KLV), Abgrenzungen zu UVG und IVG, Tarifwesen, Rechtsprechung, Leistungsfragen, Datenschutz, Kommunikation. Pro Kurs ist die Teilnehmerzahl auf 24 beschränkt, so dass sich Interessenten aufgrund der Nachfrage möglichst sofort anmelden sollten. Aufgrund der Übergangsbestimmungen müssen ja alle seit Anfang 2000 neu als VA tätigen Kollegen den WB-Kurs absolvieren. Der 1. WB-Kurs wird in deutsch durchgeführt.

Kolleginnen und Kollegen französischer und italienischer Muttersprache, welche den Kurs in französisch absolvieren wollen, sollen sich ebenfalls schon jetzt anmelden. Bei genügend Nachfrage wird ein Kurs in französischer Sprache im Jahr 2004 im Welschland durchgeführt. Mit absolviertem Weiterbildungskurs erhalten die Teilnehmer den entsprechenden Fähigkeitsausweis Vertrauensarzt.

\section{Übergangsrecht}

Gemäss Übergangsrecht erhalten alle schon vor dem 1. Januar 2000 (nebenamtliche) bzw. vor dem 1. Januar 2001 (hauptamtliche) im KVG tätigen VA den Fähigkeitsausweis, ohne die nun obligatorische Weiterbildung absolvieren zu müssen. Mitglieder der SGV werden von der Gesellschaft direkt benachrichtigt. Nichtmitglieder melden sich bei der Geschäftsstelle der SGV.

In Zukunft werden die Krankenversicherer im KVG nur noch Vertrauensärzte einsetzen können, welche über den entsprechenden Fähigkeitsausweis verfügen. Die SGV ist gewillt, dieser Regelung Nachachtung zu verschaffen. $\mathrm{Zu}$ diskutieren geben könnte auch die Bestimmung im Art. 2 des VA-Vertrages, dass der Ausdruck Vertrauensarzt für im KVG tätige Ärzte reserviert ist.

\section{Fortbildung}

Nebst der normalen Fortbildung von 80 Stunden in seinem Fachgebiet hat jeder VA zukünftig 2 Tage vertrauensarzt-relevante Fortbildung zu absolvieren. Diese kann selbstverständlich Teil dieser 80 Stunden sein. 


\section{Fähigkeitsausweis Versicherungsmedizin}

Von den in der SIM (Swiss Insurance Medicine) vertretenen Ärzten (neben der SGV umfasst diese derzeit Kollegen von SUVA, SVV, IV, MV, SGVP, ARPEM und Lebensversicherer) wird ein Fähigkeitsausweis Versicherungsmedizin angestrebt. Dieser wird möglicherweise auf den Weiterbildungskurs Vertrauensarzt aufbauen, indem zusätzliche Module geschaffen werden sollen. Form und Umfang müssen noch weiter diskutiert werden. Wünschbar ist eine solche Ausdehnung insofern, als u.a. auch die für die Unfallund Privatversicherer tätigen Gutachter ebenfalls bezüglich Versicherungsmedizin weitergebildet werden müssen. Ein weiteres Fernziel ist eine Akademie für Versicherungsmedizin, welche mithelfen soll, das diesbezügliche Vakuum an den Universitäten zu kompensieren.

\section{Paritätisches Gremium}

Der Vertrauensarztvertrag sieht ein paritätisches Gremium vor, das bei Streitigkeiten zwischen Versicherer, Leistungserbringer und Patient (und dem sich häufig zwischen Hammer und Amboss befindenden Vertrauensarzt) primär einmal den Datenschutz betreffend eingreifen kann. In diesem Gremium sind santésuisse, FMH und SGV mit je 2 Mitgliedern vertreten. Wie weit diesem Gremium zukünftig noch weitere Aufgaben zugeordnet werden sollen, wird ebenfalls noch zu diskutieren sein. Es bietet sich zumindest an, bei den so häufigen Grabenkämpfen im wörtlichen Sinn Brückenfunktion zu übernehmen. 